\title{
Morphologic and immunopathologic findings in myasthenia gravis and in congenital myasthenic syndromes
}

\author{
A N D R E G E N G L \\ From the Department of Neurology and Neuromuscular Research Laboratory, Mayo Clinic, \\ Mayo Foundation and Mayo Medical School, Rochester, Minnesota
}

SUMMARY Overwhelming evidence now supports Simpson's concept, originally proposed in 1960 , that acquired myasthenia gravis (MG) is an autoimmune disease in which antibodies are directed against the nicotine postsynaptic acetylcholine receptor (AChR). ${ }^{1}$ An autoimmune pathogenesis of acquired MG implies that those myasthenic syndromes which occur in a congenital and familial setting may have a different, non-autoimmune basis. This paper focuses on ultrastructural, immunoelectron microscopic and cytochemical aspects of acquired autoimmune MG and some recently recognised congenital myasthenic syndromes.

\section{Acquired autoimmune myasthenia gravis}

\section{Morphometric reconstruction of the ultrastructure of the motor end-plate}

These studies were carried out between 1968 and 1970 in collaboration with Tetsuji Santa. ${ }^{2}$ External intercostal muscle motor end-plates were studied from MG patients who required thymectomy, from non-weak subjects who underwent thoracotomy for intrathoracic disease and from patients with the Lambert-Eaton type of myasthenic syndrome. Problems pertaining to finding the end-plates in the electron microscope and to quantitative analysis of end-plate morphology were noted early in the study. These were overcome by devising a method for readily finding end-plates in electron microscope ${ }^{3}$ and by developing a system for the morphometric analysis of the ultrastructure of the motor end-plate. ${ }^{2}$ In this way, we were able to study a large number of end-plates in a quantitative manner and could assess the results statistically.

Our initial hypothesis was based on the then current notion that the small amplitude of the miniature end-plate potential (mepp) in MG was due to a presynaptic defect in the synthesis of acetylcholine $(\mathrm{ACh})$ or in the packaging of $\mathrm{ACh}$

Address for reprint requests: Dr AG Engel, Department of Neurology, Mayo Clinic, Rochester, Minnesota 55901, USA. into the synaptic vesicles. If this were the case, one might expect changes in the dimensions of the synaptic vesicles. Contrary to what we expected, the dimensions as well as the numerical density of the synaptic vesicles were normal. The mitochondrial fraction of the nerve terminal was also normal. However, the nerve terminals were of smaller than normal size. The normal size of the synaptic vesicles provided evidence against a decrease of the ACh content of the quantum, for when ACh synthesis is experimentally blocked by hemicholinium and the miniature end-plate potential (mepp) amplitude decreases, the synaptic vesicles become smaller.

Instead of corroborating a presynaptic defect in MG, the ultrastructural data pointed strongly to a postsynaptic abnormality. The postsynaptic regions showed degenerative changes with widening of the primary and secondary synaptic clefts and accumulation of debris in the synaptic space. Many postsynaptic regions were highly simplified and at some regions the secondary synaptic clefts were totally absent. Morphometric analysis revealed a significant decrease of the length of postsynaptic membrane and of the length of the postsynaptic membrane per unit postsynaptic area (postsynaptic membrane density). In addition, we observed sprouting of nerve terminals near endplates and encountered highly degenerated postsynaptic regions which were denuded of their 
nerve terminal. These findings were indicative of cycles of degeneration involving the postsynaptic regions.

In the Lambert-Eaton myasthenic syndrome the postsynaptic region was somewhat larger than normal and the postsynaptic membrane length was significantly increased relative to the presynaptic membrane length. There were no morphologic abnormalities of the nerve terminal and no postsynaptic degenerative changes. Morphometric reconstruction of the end-plate in control subjects, in MG and in the Lambert-Eaton syndrome is shown in fig 1. In the light of current knowledge, the postsynaptic pathology in MG is a direct consequence of the autoimmune attack on the postsynaptic membrane.

\section{Ultrastructural localisation and quantification of $A C h R$ in $M G$}

Radiochemical evidence that a deficiency of AChR existed in MG was obtained by Fambrough et al in $1973 .{ }^{4} \mathrm{My}$ co-workers and I subsequently be-
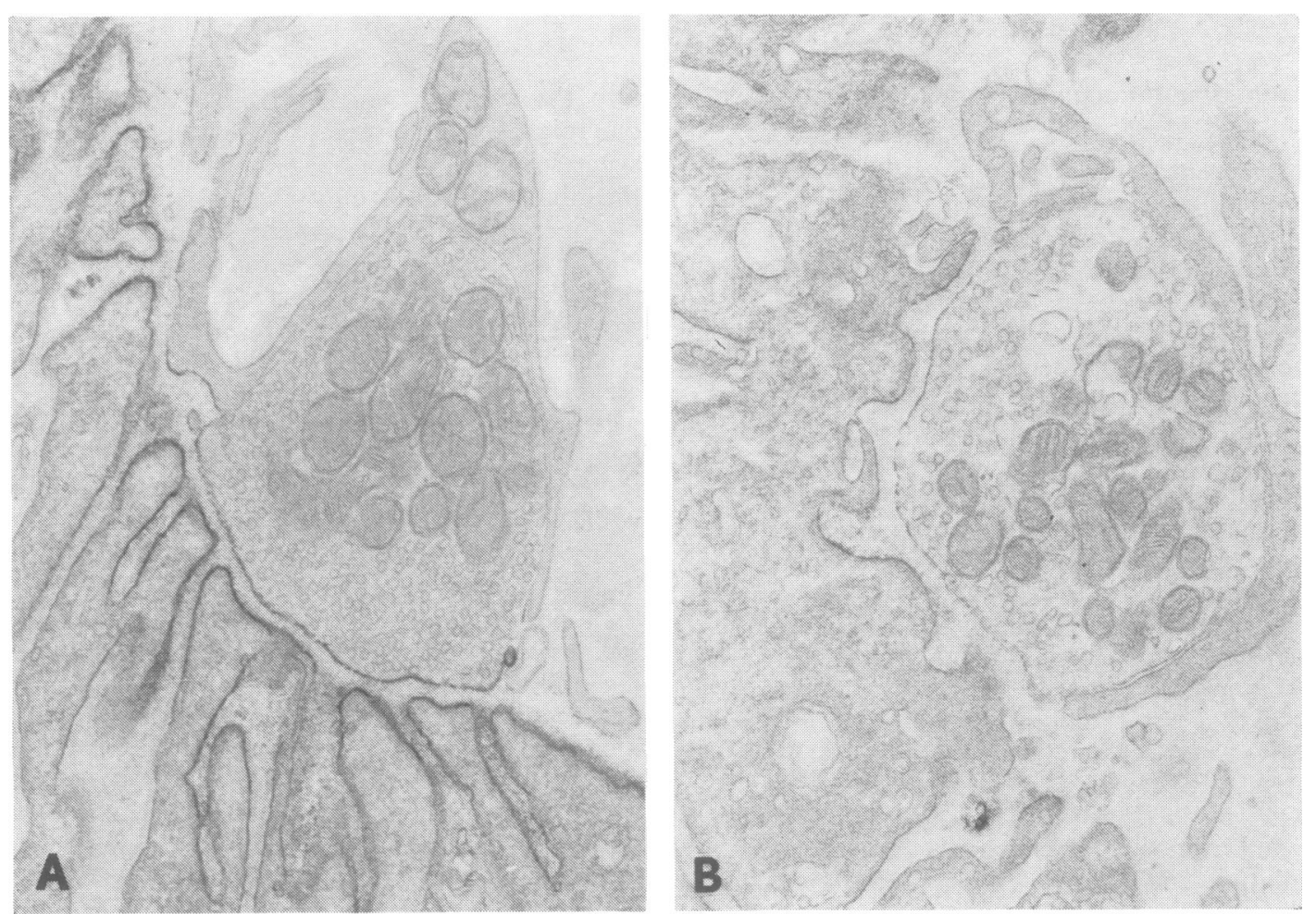

Fig 2 Ultrastructural localisation of $A C h R$ at intercostal muscle end-plate from control subject $(A)$ and in moderately severe, generalised $M G(B)$. In $B$, only short segments of simplified postsynaptic membrane react for AChR. Presynaptic staining, seen in $A$, is virtually absent in $B . A, B \times 22300$. (Reproduced from ref 5 , by permission.)
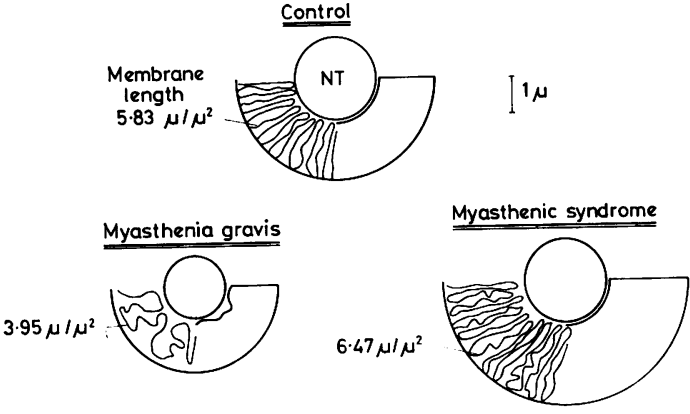

Fig 1 Morphometric reconstruction of the motor end-plate in controls, in $M G$ and in the LambertEaton syndrome. (Reproduced from ref 2, by permission.)

came interested in the ultrastructural localisation of AChR at the MG end-plate. ${ }^{56}$ Nonweak subjects and patients with the Lambert-Eaton syndrome were used for comparison. The study was facilitated by the development, in this laboratory, 
of a simple, one-stage procedure for localisation of AChR with peroxidase-labelled $\alpha$-bungarotoxin. Labelled toxin was applied to fresh, oxygenated muscle strips, well rinsed before and after exposure to the reagent, followed by glutaraldehyde fixation.

At normal end-plates, AChR was observed on terminal expansions of the junctional folds and sometimes also on stalks of the folds (fig $2 \mathrm{~A}$ ).
Less intense reaction was detected on the presynaptic membrane and occasionally on Schwann cell processes facing reactive segments of the synaptic folds. The distribution of AChR at endplates in the Lambert-Eaton syndrome resembled that seen at normal end-plates. There was deficiency of AChR at MG end-plates. In moderately severe, generalised MG the postsynaptic regions
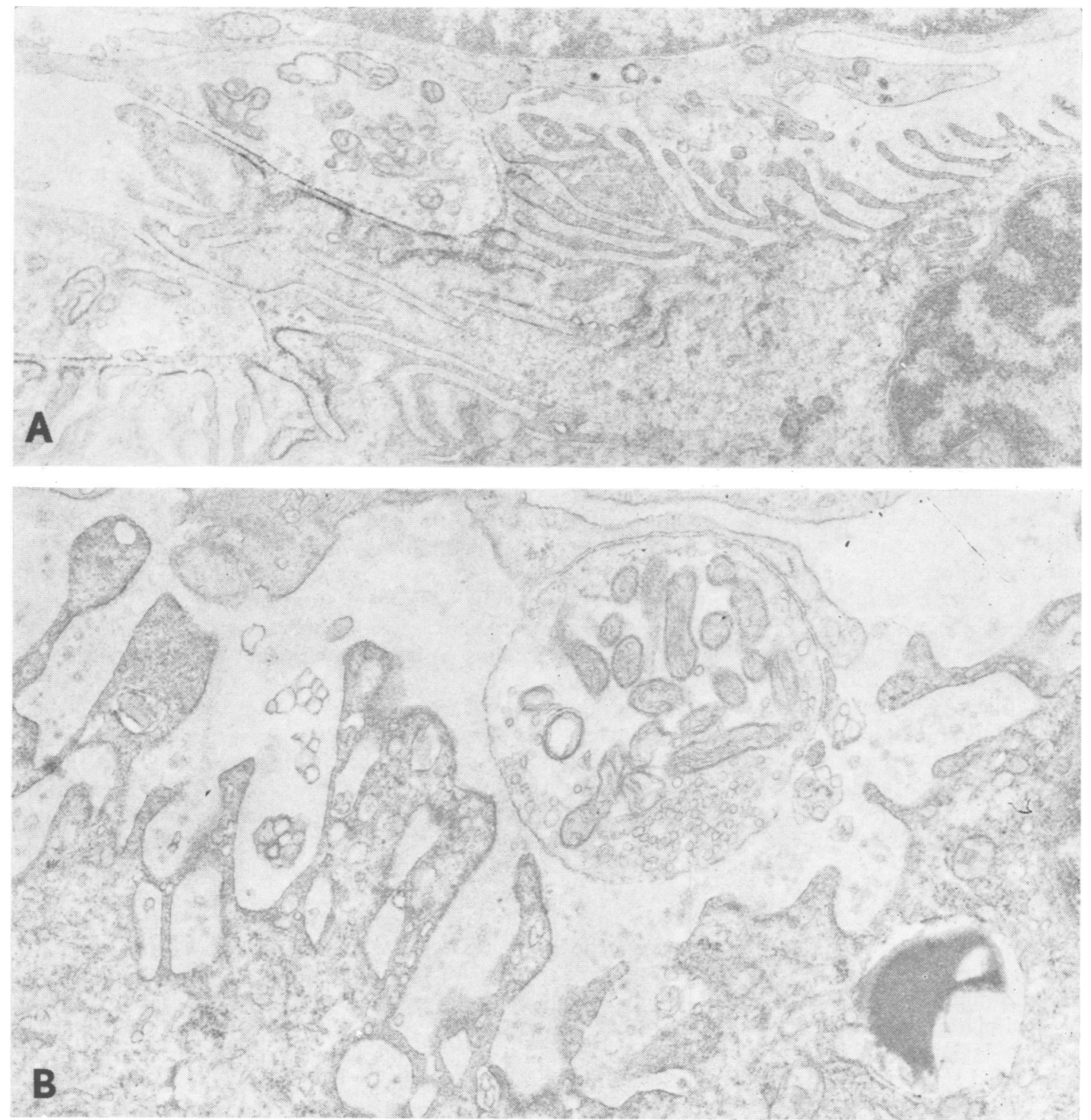

Fig 3 AChR localization at intercostal end-plate in predominantly bulbar $M G(A)$ and in moderately severe, generalised $M G(B)$. Reaction for $A C h R$ is diminished in $A$ and is undetectable in $B$. Marked degeneration of junctional folds occurs in $B$. Compare $B$ with schematised $M G$ end-plate in fig 1 . $A, \times 17$ 900; $B, \times 22300$. (Reproduced from ref 5, by permission.) 
usually showed a marked decrease (fig 2B), or even absence (fig 3B), of $A C h R$. In less severe MG, AChR seemed normally abundant at some endplate regions but was diminished at others (fig $3 \mathrm{~A}$ ). Usually, but not always, the simplest and most degenerated postsynaptic regions showed the greatest decrease in toxin binding. As a rule, the presynaptic staining was reduced or absent when vis-à-vis junctional folds did not react for AChR (figs $2 \mathrm{~B}$ and $3 \mathrm{~B}$ ). This suggested that the presynaptic staining was largely an artifact caused by diffusion of reaction product from postsynaptic sites.

To quantify the observations, the length of the postsynaptic mombrane reacting for AChR at a given end-plate region was measured and then normalised for the length of the presynaptic cleft. The value thus obtained was an index of the relative abundance of the postsynaptic AChR for that region. In the MG patients the mean AChR index ranged from 0.27 to 1.62 (group mean \pm SEM: $0 \cdot 85 \pm 0 \cdot 14$ ). In the nonweak controls the corresponding values ranged from 3.06 to 3.29 (3.21 \pm 0.05$)$; and in the Lambert-Eaton syndrome from $2 \cdot 56$ to $3 \cdot 77(3.05 \pm 0 \cdot 16)$.

In collaboration with Dr EH Lambert, we attempted to correlate the morphometric data with in vitro electrophysiologic observation. ${ }^{56}$ To this end, the mean AChR indices obtained in each subject were compared with the corresponding mean mepp amplitudes. The comparison demonstrated a linear correlation between the two variables $(r=0.899 ; p \ll 0.001)$.

These observations in MG provided unambiguous ultrastructural evidence for a deficiency of postsynaptic AChR and indicated that the deficiency of AChR determined the small mepp amplitude. A linear relationship between the AChR content of muscle and the mepp amplitude was also noted in subsequent studies in which AChR was radiochemically estimated. ${ }^{7} 8$

\section{Ultrastructural localisation of $\operatorname{IgG}$ at the $M G$ end-plate}

Although evidence for an autoimmune pathogenesis of acquired MG had been accumulating since 1973 , no immunoglobulin was demonstrated at the MG end-plate before 1977. We succeeded in localising IgG at the MG end-plate with peroxidase-labelled staphylococcal protein A. ${ }^{9}$ Protein A binds bivalently to $F c$ portions of human IgG subclasses 1,2 and 4 and has an increased affinity for IgG molecules attached to antigenic sites. ${ }^{10}$ The latter property enhances the specificity and sensitivity of the reagent and optimises the signal (reaction at the end-plate) to noise (background staining) ratio of the cytochemical procedure.

As in the preceding study, the immunoreagent was applied to fresh, intact muscle strips, well rinsed before and after the application of the immunoreagent. In less severe cases of $\mathbf{M G}$, in which the postsynaptic folds were relatively well preserved, IgG was found over terminal expansions of the junctional folds where AChR is known to be located (figs 4A and C). In more severe cases, in which junctional folds were less well preserved, the postsynaptic membrane bound less IgG, but IgG was readily detected on degenerated remnants of the junctional folds in the synaptic space (fig 4B). Morphometric estimates of the abundance of the postsynaptic membrane binding IgG were proportionate to the mepp amplitude, but an exception to this relationship was noted in one of ten cases. This was a young patient who, despite having moderately severe disease, had well preserved junctional folds which bound abundant IgG.

From these findings we inferred that (1) the amount of IgG bound to the postsynaptic membrane is proportionate to the amount of AChR which remains in the membrane; (2) IgG-coated segments of junctional folds are shed into the synaptic space; (3) in most cases of MG the transmission defect is determined by the loss of AChR rather than by the fact that IgG is bound to AChR; (4) in most cases of MG, antibodies to AChR do not significantly block the response of AChR to ACh, but in occasional patients antibodies do have a significant blocking effect.

Participation of the complement system in the autoimmune attack on the postsynaptic membrane We sought evidence for antibody dependent complement mediated injury to the junctional folds by localising complement components at the MG end-plate. ${ }^{611}$ The third (C3) and ninth (C9) complement components were localised with monospecific antibodies raised in rabbits. The ultrastructural localisation of $\mathrm{C} 3$ was identical to that of IgG. Reaction product was detected on segments of the postsynaptic membrane, on debris in the synaptic space and on disintegrating junctional folds. ${ }^{9}$ The fact that the distributions of $\mathrm{C} 3$ and IgG at the end-plate are highly similar is consistent with the assumption that anti-AChR antibodies can fix complement and that the assembly phase of the complement reaction sequence (via $\mathrm{C} 1, \mathrm{C} 4$, $\mathrm{C} 2$ and $\mathrm{C} 3$ ) has gone to completion. However, the presence of $\mathrm{C} 3$ on the junctional folds does not in itself establish that the attack (or lytic) phase of 

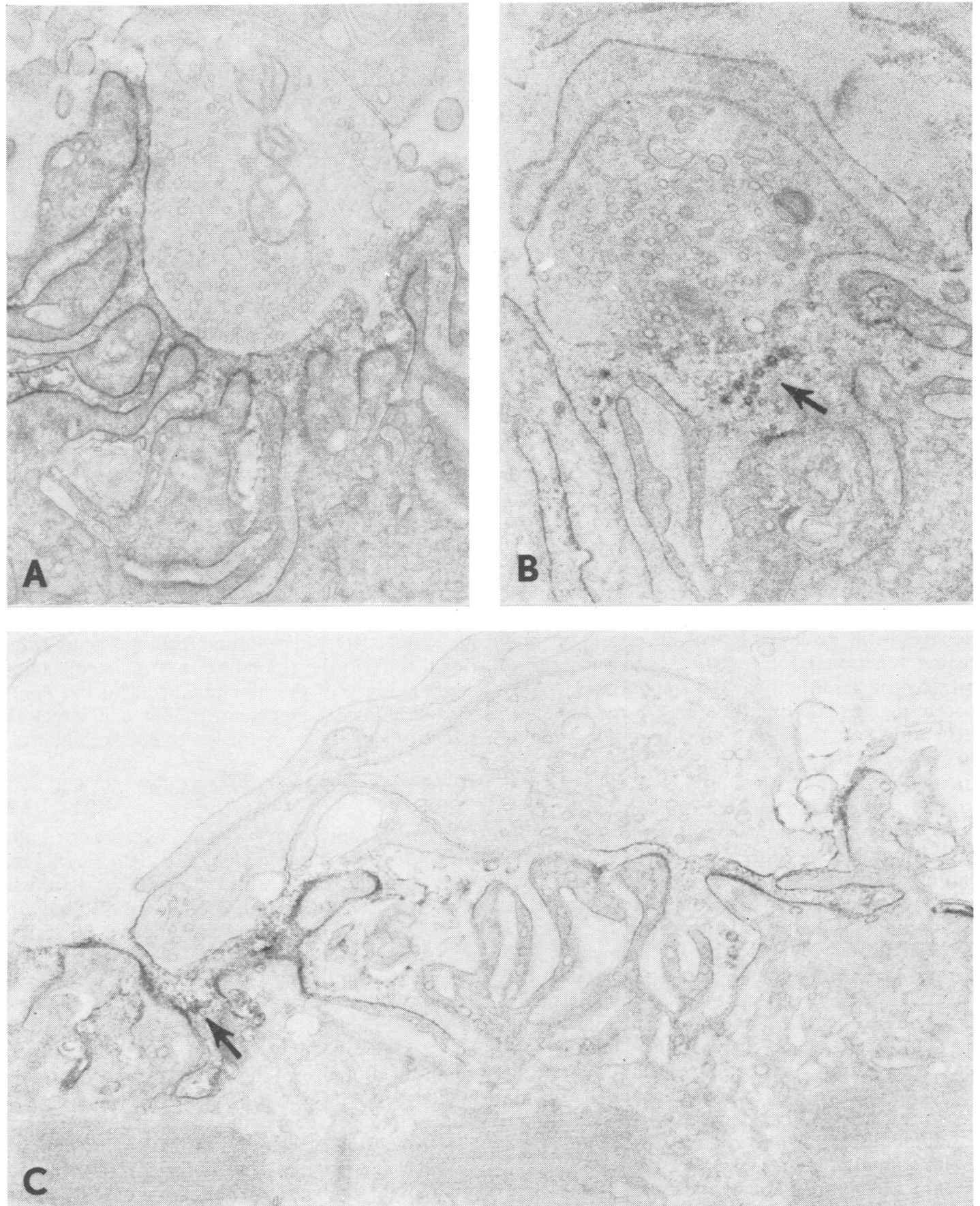

Fig 4 Ultrastructural localisation of $\operatorname{IgG}$ at the $M G$ end-plate. $\operatorname{Ig} G$ is detected on terminal expansions of well preserved junctional folds $(A)$, on degenerate material shed into synaptic space (arrows in $B$ and $C$ ), and on short segments of the postsynaptic membrane $(B$ and $C)$. Note that in $C$ presynaptic staining is reciprocal to postsynaptic reaction. $A, \times 25100 ; B, \times 29700 ; C, \times 24800$. (Reproduced from ref 9 by permission.) 
the complement reaction has become activated. ${ }^{12}$

The occurrence of complement mediated membrane injury depends on the presence on the target membrane of C5b, C6, C7, C8 and C9, the constituents of the membrane attack complex. The complex, now known to be a dimer of C5b-9, induces focal and irreversible membrane lesions and C9 is an absolute requirement for this. ${ }^{12} 13$ Therefore, to obtain unambiguous evidence for complement mediated injury of the postsynaptic membrane, we decided to study the localisation of C9 at the MG end-plate. ${ }^{11}$

At end-plates where the architecture of the postsynaptic region was well preserved, C9 was localised on short segments of the junctional folds and on sparse debris in the synaptic space. At endplates which showed degenerative changes, there was intense reaction for $\mathrm{C} 9$ on degenerate material in the synaptic space and this was most marked where the junctional folds were the most abnormal (fig 5A). Reaction for C9 also occurred near existing end-plates, on debris positioned between layers of basal lamina. Finally, intense reaction for C9 was found at highly degenerate postsynaptic regions denuded of their nerve terminal (fig 5B). Here the reaction was on debris which often was still arranged in the shape of the preexisting junctional fold and was still surrounded by traces of basal lamina. On some muscle fibres discrete regions reacting for $\mathrm{C} 9$ were widely separated. Some of these were postsynaptic regions covered by nerve terminal; some consisted of degenerate folds and debris not covered by nerve terminal; and some consisted only of debris sandwiched between layers of basal lamina (fig 5C). In contrast with the localisation of IgG and C3, no definite relationship could be established between the abundance of the reaction for C9 (most of which was associated with debris) and the severity of the disease or the amplitude of the mepp.

From these findings we inferred that (1) the presence of IgG or C3 on the postsynaptic membrane does not in and of itself injure the junctional folds; (2) the complement reaction sequence, subject to constraints of time and space, does not go to completion at all sites that had bound C3; (3) once formed, the C5b-9 dimer is more stable than the membrane which it attacks; (4) segments of the junctional folds attacked by the C5b-9 complex are shed into the synaptic space where they further disintegrate; (5) debris labelled by the C5b-9 complex is tell-tale evidence of previous, repeated or ongoing complement attack on the end-plate; (6) remodelling of the end-plate and progressive separation of end-plate regions occur when the nerve terminal moves away from junctional folds destroyed by complement to an adjacent site where a new end-plate region develops.

Complement mediated lysis of the junctional folds represents a plausible mechanism for loss of AChR from the postsynaptic membrane in human MG. Accelerated internalisation and intracellular disposal of AChR molecules cross-linked by IgG (modulation) might also contribute to the receptor deficiency at the end-plate. ${ }^{14-16}$ From the available evidence, the relative significance of modulation versus shedding (internalisation versus externalisation) of $\mathrm{AChR}$ cannot be ascertained. Both mechanisms may well play a role in causing AChR deficiency. It should be noted, however, that modulation of AChR by antibody will not entail structural damage to the postsynaptic membrane. Thus, if modulation alone were responsible for the AChR deficiency, one would not expect to see structural changes at the MG end-plate. However, such changes do occur. Finally, there is as yet no immunoelectron microscopic corroboration of modulation in human MG.

Focal degeneration of the junctional folds can also occur in Duchenne dystrophy and in certain congenital myasthenic syndromes. However, we have not detected immune complexes on junctional folds in these conditions. Thus, complement mediated lysis of the junctional folds is but one of the pathological mechanisms that can affect the structural integrity of the postsynaptic region.

\section{Congenital myasthenic syndromes}

\section{Congenital familial myasthenic syndrome}

attributed to a putative defect of $A C h$ resynthesis

This disorder was studied in collaboration with Zwi Hart, Ko Sahashi, EH Lambert and Jon Lindstrom. ${ }^{17}$ The clinical features of the family we studied were similar to those described in "familial infantile myasthenia" in the literature. ${ }^{18}$ These included fluctuating ptosis since birth, feeding difficulty during infancy, easy fatigability on exertion and episodic apnea following crying, vomiting or febrile illnesses. No circulating antibodies to AChR were detected. This disease is transmitted by autosomal recessive inheritance. Electrophysiologic studies by EH Lambert revealed a decremental response at $2 \mathrm{~Hz}$ stimulation but only after exercise. The mepp amplitude was normal in rested muscle but decreased to abnormally low values after $10 \mathrm{~Hz}$ stimulation for a few minutes.

Electron microscopic examination of motor endplates in rested muscle showed no qualitative ab- 

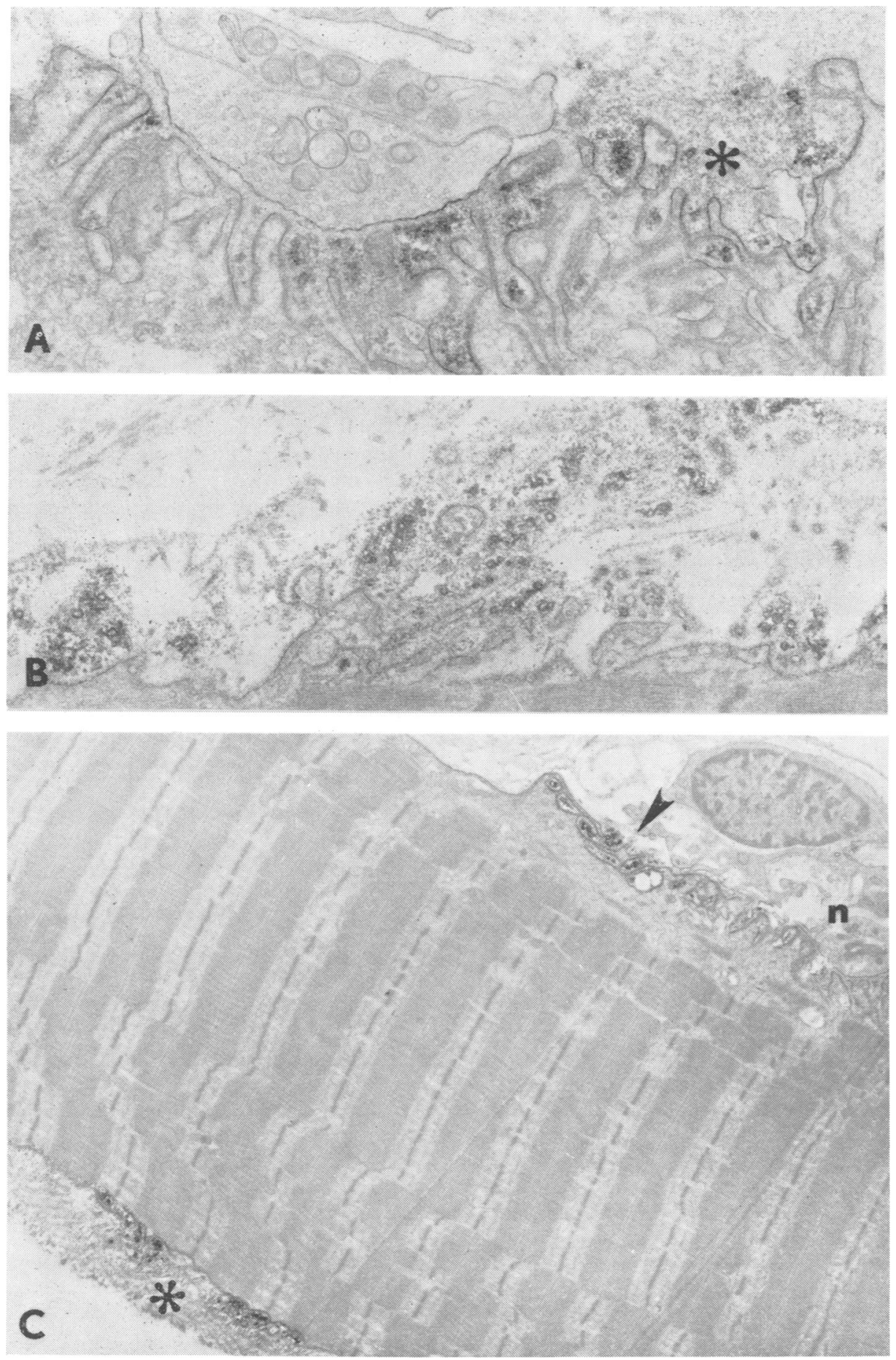

Fig 5 Ultrastructural localisation of $C 9$ at the $M G$ end-plate. In $A, C 9$ positive debris is accumulating in widened synaptic space on the right. Shortest folds are not covered by nerve terminal (asterisk). At the left, junctional folds are well preserved and only sparse $C 9$-positive material appears between them. B shows highly degenerated postsynaptic region with disintegrating folds composed of C9-positive debris covered by basal lamina. This postsynaptic region is denuded of its nerve terminal. In C, reaction for C9 occurs on debris where an end-plate region previously existed (asterisk) and at the opposite side of fibre near degenerating junctional folds (arrow). Nerve terminal $(n)$ is seen to right of folds.

$A, \times 16800 ; B, \times 12900 ; C, \times 5500$. (Reproduced from ref 11, by permission.) 
normality. Morphometric reconstruction of the motor end-plate was based on analysis of 122 regions in 55 end-plates. The size of the nerve terminals was normal. Synaptic vesicles were of normal size but their numerical density was 60 per cent greater than normal. The postsynaptic area of folds and clefts and the postsynaptic membrane length were normal. Ultrastructural localisation of AChR showed a normal abundance and distribution of the receptor on terminal expansions of the junctional folds and a normal AChR index. Immunoelectron microscopic studies revealed no IgG or C3 at the end-plate. Acetylcholinesterase activity was normal by light microscopic criteria.

It is clear that the neuromuscular transmission defect in this disorder cannot be attributed to a deficiency of AChR. The fact that an abnormally small mepp amplitude was found only after prolonged stimulation suggests a defect in ACh resynthesis. This might be related to a defect in the facilitated reuptake of choline by the nerve terminal, a deficiency of choline acetyltransferase, or possibly an abnormality in the packaging of quanta into the synaptic vesicles. Additional ultrastructural studies, including examination of the endplate in stimulated muscle when neuromuscular transmission fails, and biochemical investigations will be required to establish the nature of the basic abnormality.

Congenital myasthenic syndrome associated with end-plate acetylcholinesterase ( $A C h E)$ deficiency, small nerve terminals and reduced $A C h$ release

This disorder was investigated in collaboration with EH Lambert and MR Gomez. ${ }^{18}$ The patient was first observed by us at age $14 \mathrm{yr}$. His symptoms began soon after birth and included fluctuating ptosis, intermittent strabismus, delayed motor development, generalised weakness increased by exertion, easy fatigability, hyporeflexia and refractoriness to anticholinesterase drugs. No other family members were similarly affected. Electrophysiologic studies by EH Lambert showed a decremental response at all frequencies of stimulation and a repetitive muscle action potential response to single nerve stimulus. In three muscle specimens, mepp's were of normal amplitude but of decreased frequency. The mepp duration and half-decay times were markedly prolonged and prostigmine was without additional effect. The quantum content of the end-plate potential (epp) at $1 \mathrm{~Hz}$ stimulation was decreased due to a re-
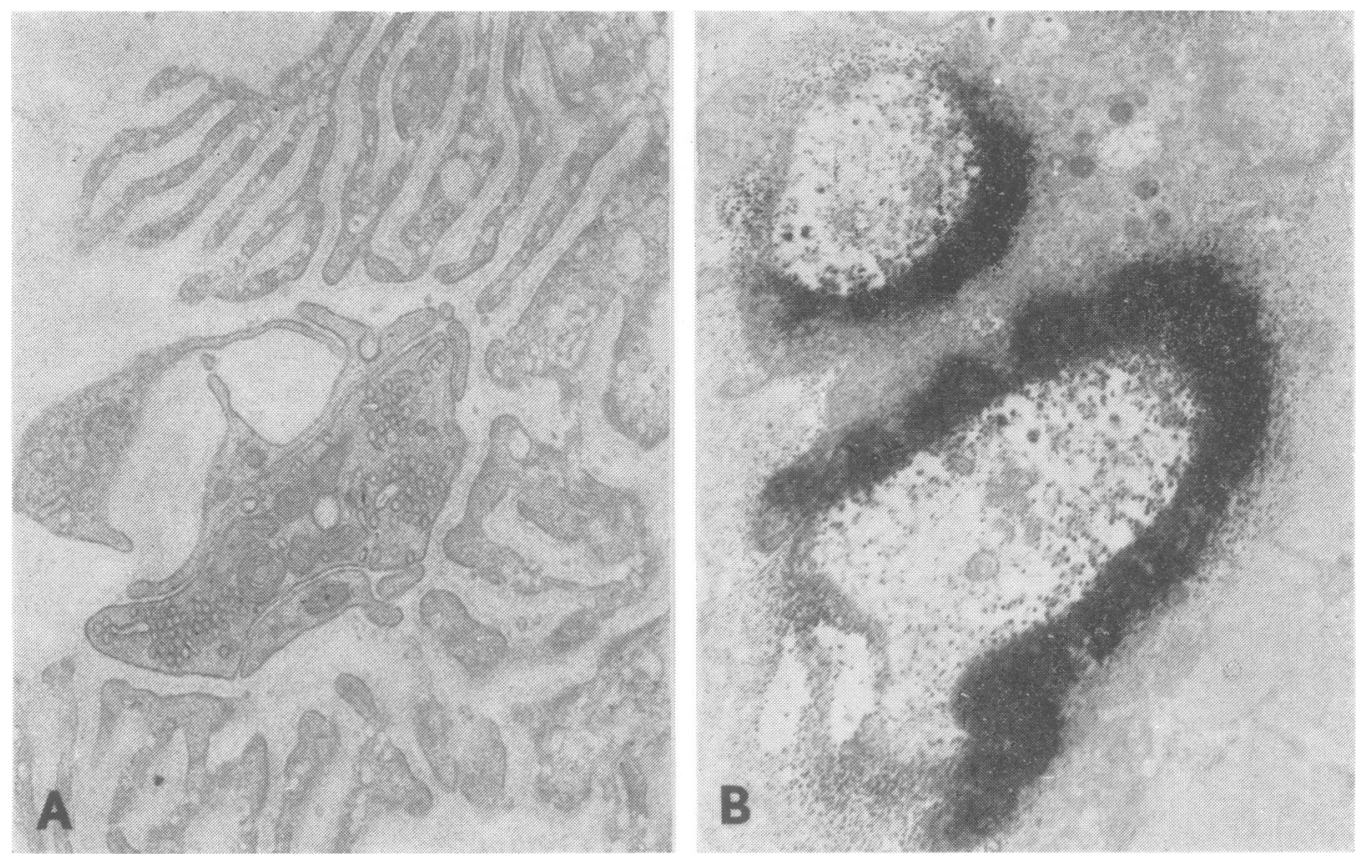

Fig 6 AChE localisation in patient with $A C h E$ deficiency $(A)$, and in control subject $(B)$. At the patient's end-plate there is no reaction after 1 hour of incubation at room temperature. Control end-plate is greatly overreacted after 30 minutes incubation at same temperature. A, $\times 20500$; $B, \times 8900$. (Reproduced from ref 19 , by permission.) 

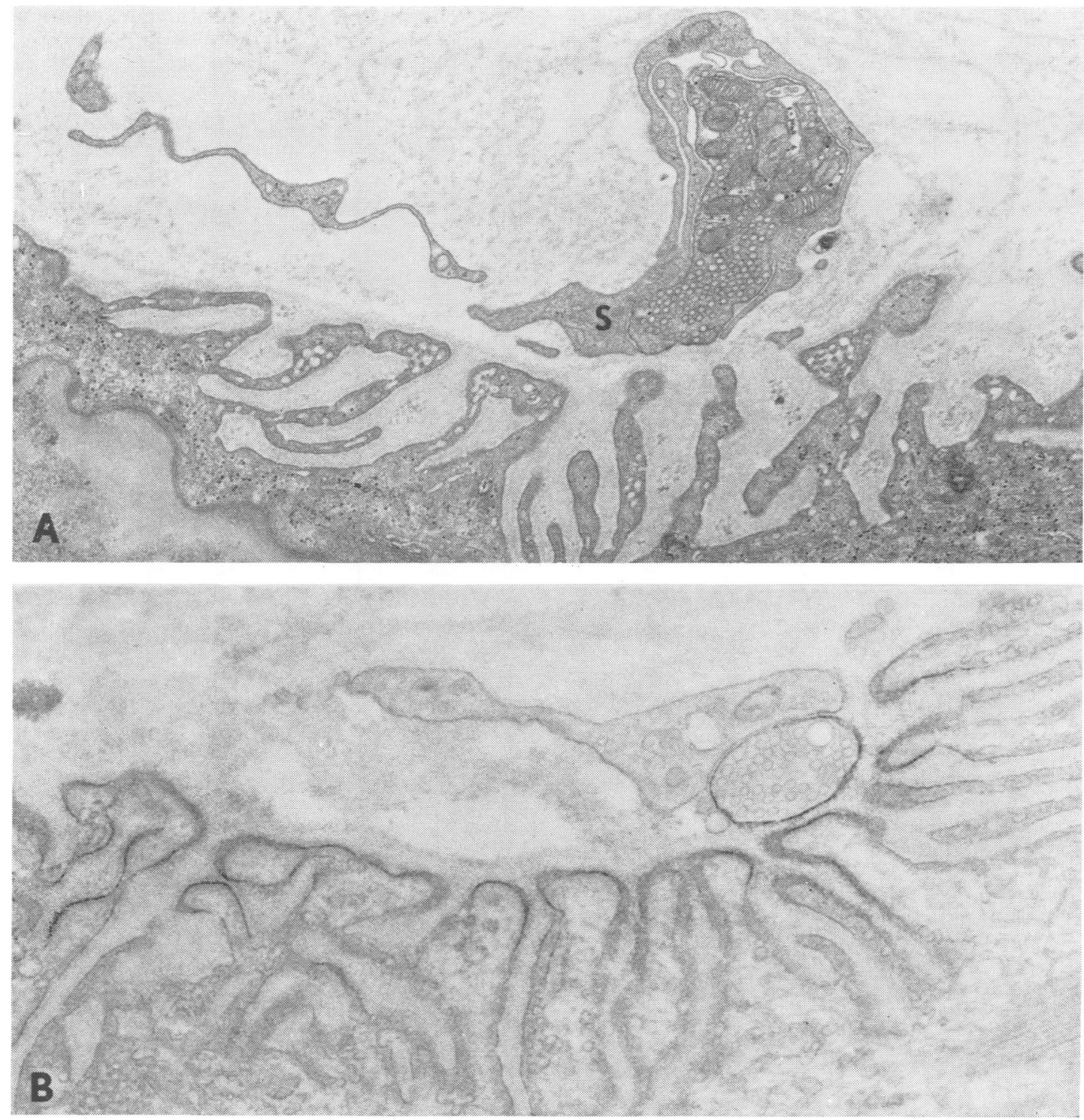

Fig 7 Congenital end-plate AChE deficiency. In A, small nerve terminal, almost completely surrounded by Schwann cell $(S)$, is applied against a small fraction of the postsynaptic region. The junctional folds contain numerous membranous networks. B shows normal abundance and distribution of AChR on terminal expansions of junctional folds. Nerve terminal is small, as in A. A, $\times 15400 ; B, \times 23400$. (Fig 6 A is reproduced from ref 19 , by permission.)

duced store of quanta immediately available for release, but the probability of release was normal.

Routine histochemical studies revealed no abnormalities of the muscle fibre but there was total absence of AChE from all motor end-plates by histochemical and electron cytochemical (fig 6) criteria. Sucrose density gradient ultracentrifuga- tion of AChE extracted from the patient's muscle showed a virtual absence of the $16 \mathrm{~S}$ species of the enzyme and a marked decrease in total AChE. Although AChE was completely absent from all end-plates, AChR was readily demonstrated at the end-plates with peroxidase labelled $\alpha$-bungarotoxin and the ultrastructural localisation of AChR 
was normal (fig 7B). The nerve terminals were generally small (fig 7) and quantitative electron microscopy revealed a three to fourfold decrease of nerve terminal size and a significant increase in the numerical density of synaptic vesicles. Some postsynaptic folds showed focal degeneration and many were distended by labyrinthine membranous networks that communicated with the synaptic space (fig 7A).

The absence of end-plate AChE readily explains the patient's refractoriness to anticholinesterase drugs, the repetitive muscle action potential to
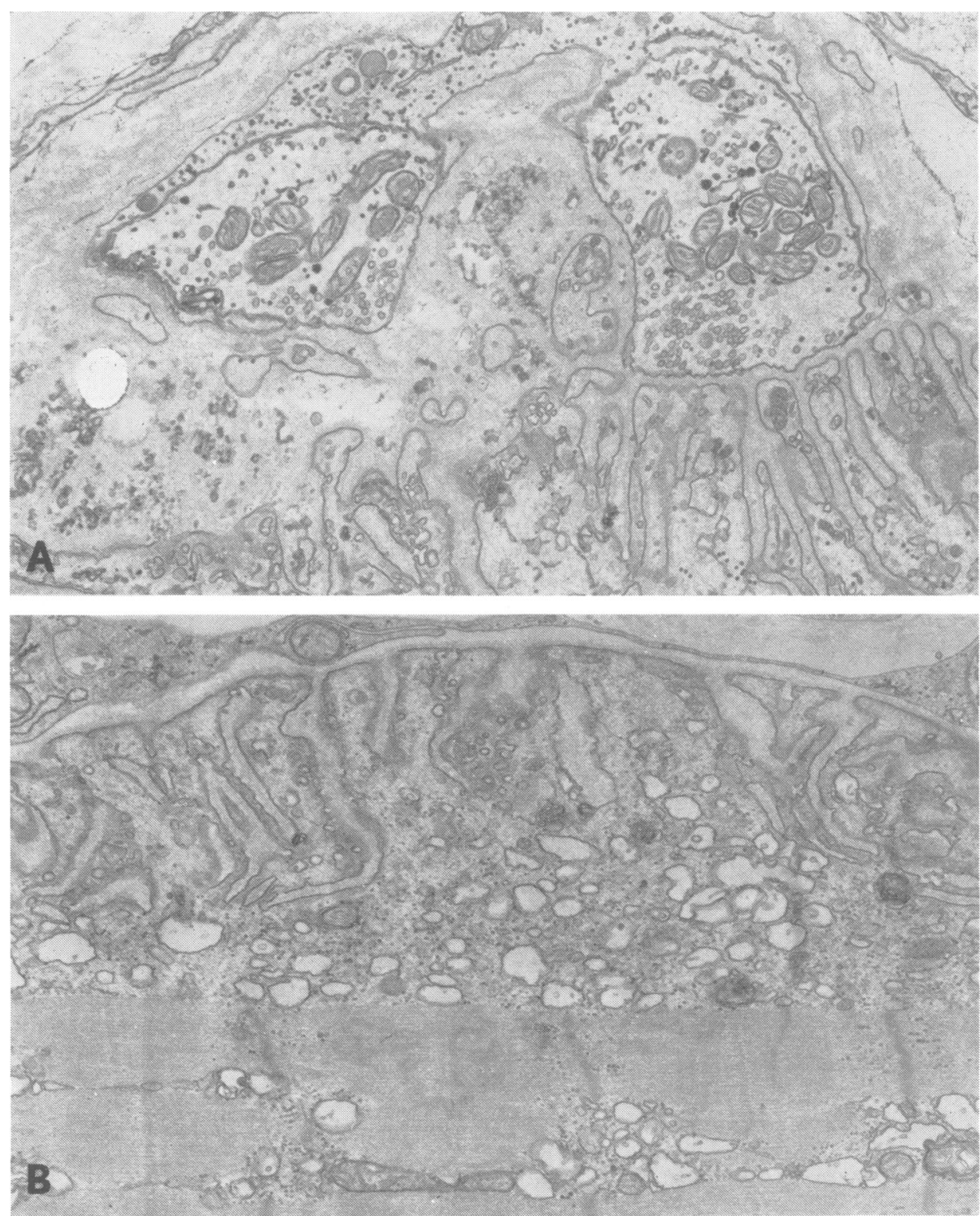

Fig 8 Putative defect of ACh induced ion channel. In A, all junctional folds have degenerated in end-plate region imaged at left. Numerous endocytotic vesicles appear in preserved junctional folds on right. In $B$, junctional sarcoplasm and underlying fibre region contain numerous dilated vesicles of the sarcoplasmic reticulum. $A, \times 15500 ; B, \times 19000$. 
single nerve stimulus, ${ }^{20}$ the abnormal duration and prolonged half-decay time of the mepp, ${ }^{21}$ and the failure of prostigmine to have any additional effect on the mepp in vitro. The decreased quantum content of the epp in the presence of a normal probability of release is adequately accounted for by the smallness of the nerve terminal. ${ }^{22}$ The physiological and clinical phenomena in this case can be attributed to two findings: the end-plate AChE deficiency and the smallness of the nerve terminals. Of these, the enzyme deficiency is probably primary and smallness of the nerve terminal compensatory, or secondary. This assumption is supported by the fact that AChE was monotonously absent from all end-plates whereas the decrease in nerve terminal size was statistical, with some terminals of normal size. An alternative possibility would be a primary, but yet undefined, presynaptic disturbance which resulted in small nerve terminals and defective neural regulation of end-plate AChE.

Familial congenital myasthenic syndrome atrributed to a putative defect of the ACh induced ion channel

This disorder was studied in collaboration with Drs EH Lambert, DW Mulder, K Sahashi, CF Torres, TE Bertorini and JN Whitaker. ${ }^{23}$ The disease presents in childhood or adult life, pro- gresses slowly and is associated with variable weakness of ocular, facial, cervical and masticatory muscles, and with selectively severe weakness and atrophy of scapular and forearm muscles. In the familial cases there was an autosomal dominant pattern of inheritance. Electrophysiologic studies by EH Lambert showed a repetitive muscle action potential to single nerve stimulus in all muscles and a decremental response to 2 to $3 \mathrm{~Hz}$ stimulation of clinically affected muscles. Microelectrode studies revealed markedly prolonged epp's and mepp's which became even more prolonged on addition of prostigmine, normal quantum content of the epp and a smaller than normal or low normal mepp amplitude.

Light microscopic histochemical studies showed type 1 fibre predominance, isolated or small groups of atrophic fibres of either histochemical type, tubular aggregates and vacuoles in fibre regions near motor end-plates, abnormal variation in fibre size, variable fibre splitting and, in some biopsies, mild to moderate increase of perimysial connective tissue. Abundant AChE activity was present at all motor end-plates. Immunoelectron microscopic studies revealed no IgG, C3 or C9 at the motor end-plate. Quantitative electron microscopy of 132 end-plates in three cases demonstrated 30 to 40 per cent decrease of nerve terminal size, an increased number of synaptic vesicles and re-

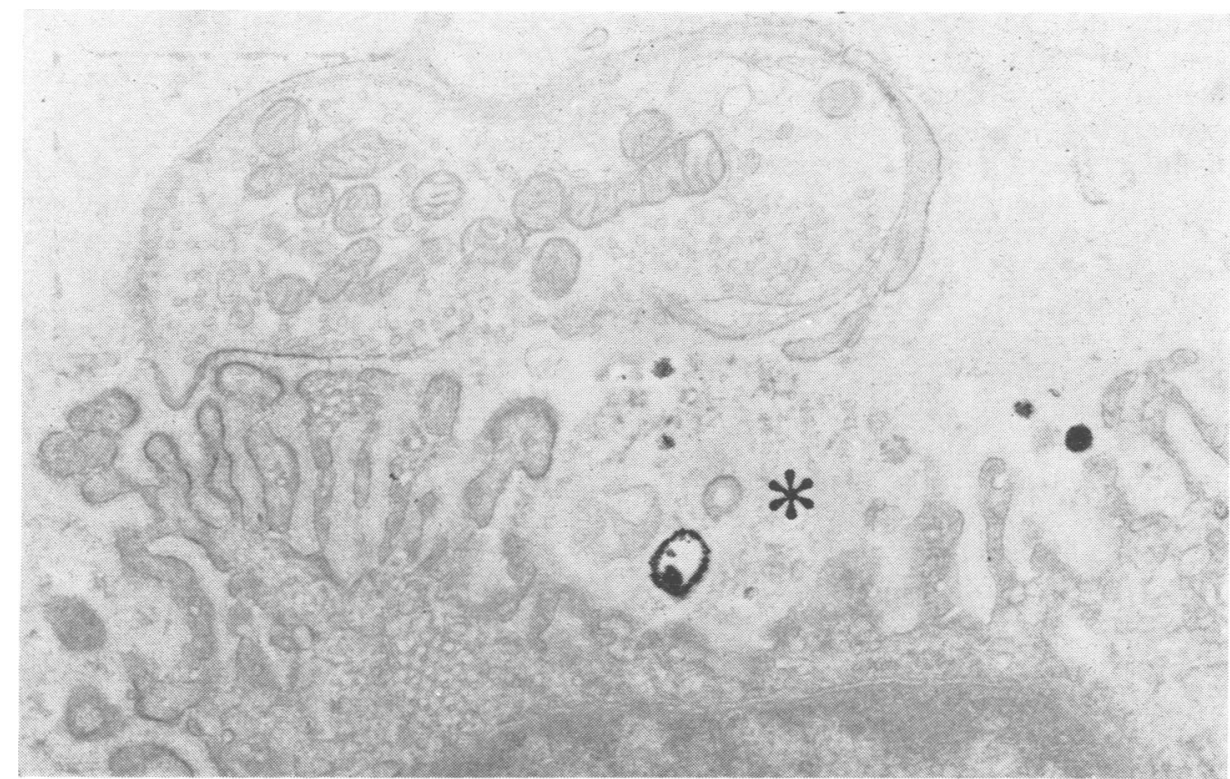

Fig $9 A C h R$ localisation in patient with putative defect of the ACh induced ion channel. $A C h R$ is lost where junctional folds have degenerated (asterisk). Circular, dense calcific deposits occur in remnants of folds. $\times 20000$. 
duced postsynaptic membrane length. There was focal degeneration of junctional folds (figs $8 \mathrm{~A}$ and 9) associated with loss of AChR (determined with peroxidase-labelled $\alpha$-bungarotoxin), most marked in the case with the lowest mepp amplitude (fig 9). In addition, many postsynaptic regions had one or more of the following abnormalities: myriad pinocytotic vesicles and abundant labyrinthine membranous networks in the junctional folds (figs 8A and 9); dilated sarcoplasmic reticulum components in fibre regions near the end-plates (fig 8B); and focal cytoplasmic degradation and autophagic vacuoles in the junctional region.

The repetitive muscle action potential in this syndrome is attributed to the prolonged epp. The latter could be due to a prolonged presence of $\mathrm{ACh}$ in the synaptic space, as when AChE is inhibited, ${ }^{21}$ AChE deficiency (as in the previous syndrome), or a prolonged open time of the $\mathrm{ACh}$ induced ion channel. ${ }^{24}$ The last explanation is the most likely because our patients were not exposed to anticholinesterase drugs and abundant AChE was present at all end-plates. The ion channel abnormality must be present in all muscles but the transmission defect and weakness occur only in selected muscles. On the basis of the morphologic findings, the transmission defect is attributed to degeneration of the junctional folds with concomitant loss of AChR. The degeneration may be secondary to the abnormal subsynaptic ionic milieu caused by the prolonged open time of the receptor ion gate. Finally, the alterations in muscle fibre architecture and innervation may also be secondary to the abnormal interaction between ACh and its receptor.

\section{Other congenital myasthenic syndromes}

It is entirely conceivable that additional myasthenic syndromes will be defined by combined clinical, ultrastructural, electrophysiological and biochemical studies. Hypothetically, there might be genetically determined abnormalities of the AChR macromolecule which could decrease its affinity for $\mathrm{ACh}$, or result in a diminshed conductance or open time of the ACh induced ion channel. Mutations involving cytoskeletal proteins might cause faulty insertion of AChR into the postsynaptic membrane or accelerated removal of AChR from the membrane. Further, any mutation affecting the structure of the synaptic vesicles, the presynaptic membrane or the junctional folds could adversely affect the safety margin of neuromuscular transmission. Thus, future studies of the congenital myasthenic syndromes, as well as of acquired autoimmune MG, may prove to be challenging and rewarding to clinicians and researchers alike.

Work in the author's laboratory was supported in part by Research Grant NS6277 from the National Institutes of Health and by a Research Centre Grant from the Muscular Dystrophy Association.

\section{References}

1 Simpson JA. Myasthenia gravis: A new hypothesis. Scot Med J 1960; 5:419-36.

2 Engel AG, Santa T. Histometric analysis of the ultrastructure of the neuromuscular junction in myasthenia gravis and in the myasthenic syndrome. Ann NY Acad Sci 1971; 183:46-63.

3 Engel AG. Locating motor end-plates for electron microscopy. Mayo Clin Proc 1970; 45:450-4.

4 Fambrough DM, Drachman DB, Satyamurti S. Neuromuscular junction in myasthenia gravis: decreased acetylcholine receptors. Science 1973; 182:293-5.

5 Engel AG, Lindstrom JM, Lambert EH, Lennon VA. Ultrastructural localization of the acetylcholine receptor in myasthenia gravis and in its experimental autoimmune model. Neurology (Minneap.) 1977; 27:307-15.

6 Engel AG, Sahashi K, Lambert EH, Howard FM. The ultrastructural localization of the acetylcholine receptor, immunoglobulin $G$ and the third and ninth complement components at the motor end-plate and their implications for the pathogenesis of myasthenia gravis. In: Aguayo AJ, Karpati G, eds, Current Topics in Nerve and Muscle Research 1979; Amsterdam-Oxford: Excerpta Medica, ICS 455:111-22.

7 Lindstrom J, Lambert EH. Content of acetylcholine receptor and antibodies bound receptor in myasthenia gravis, experimental autoimmune myasthenia gravis, and Eaton-Lambert syndrome. Neurology (Minneap.) 1978; 28:130-8.

8 Ito $\mathrm{Y}$, Miledi R, Vincent A, Newsom-Davis J. Acetylcholine receptors and end-plate electrophysiology in myasthenia gravis. Brain 1978; 101: 345-68.

9 Engel AG, Lambert EH, Howard FM. Immune complexes (IgG and C3) at the motor end-plate in myasthenia gravis. Ultrastructural and light microscopic localization and electrophysiologic correlations. Mayo Clin Proc 1977; 52:267-80.

10 Kessler SW. Cell membrane antigen isolation with the staphylococcal protein-A antibody absorbent. J Immunol 1976; 117:1482-90.

11 Sahashi K, Engel AG, Lambert EH, Howard FM. Ultrastructural localization of the terminal and lytic ninth complement component (C9) at the motor end-plate in myasthenia gravis. $J$ Neuropathol Exp Neurol 1980; In press.

12 Müller-Eberhard HJ. Complement. Annu Rev Biochem 1975; 44:697-724. 
13 Biesecker G, Podack ER, Halverson CA, MüllerEberhard HJ. C5b-9 dimer: isolation from complement-dependent membrane lesions. J Exp Med 1979; 149:448-58.

14 Reiness GC, Weinberg CB, Hall ZW. Antibody to acetylcholine receptor increases degradation of junctional and extrajunctional receptor in adult muscle. Nature 1978; 274:68-70.

15 Heinemann S, Merlie J, Lindstrom J. Modulation of acetylcholine receptor in rat diaphragm by antireceptor sera. Nature 1978; 274:65-8.

16 Stanley EF, Drachman DB. Effect of myasthenic immunoglobulin an acetylcholine receptors of intact mammalian neuromuscular junctions. Science 1978; 200:1285-7.

17 Hart Z, Sahashi K, Lambert EH, Engel AG, Lindstrom J. A congenital familial myasthenic syndrome caused by a presynaptic defect of transmitter resynthesis or mobilization. Neurology (Minneap.) 1979; 29:559.

18 Robertson WC, Chun RWM, Kornguth SE. Familial infantile myasthenia. Arch Neurol 1980; 37:117-9.

19 Engel AG, Lambert EH, Gomez MR. A new myasthenic syndrome with end-plate acetylcholinesterase deficiency, small nerve terminals and reduced acetylcholine release. Ann Neurol 1977; 1:315-30.

20 Brown GL, Dale HH, Feldberg W. Reaction of the normal mammalian muscle to acetylcholine and eserine. J Physiol (Lond) 1936; 87: $394-424$.

21 Katz B, Miledi R. The binding of acetylcholine to receptors and its removal from the synaptic cleft. J Physiol (Lond) 1973; 231:549-74.

22 Bennett MR, Florin TJ. A statistical analysis of the release of acetylcholine at newly formed synapses in striated muscle. J Physiol (Lond) 1974; 238:93-107.

23 Engel AG, Lambert EH, Mulder DW, Torres CF, Sahashi K, Bertorini TE, Whitaker JN. Investigations of 3 cases of a newly recognized familial, congenital myasthenic syndrome. $A n n$ Neurol 1979; 6:146.

24 Anderson CR, Stevens CF. Voltage clamp analysis of acetylcholine produced end-plate current fluctuations at frog neuromuscular junction $J$ Physiol (Lond) 1973; 235:655-91. 